UVX 2012, 01017 (2013)

DOI: $10.1051 /$ uvx/201301017

(C) Owned by the authors, published by EDP Sciences, 2013

\title{
Microcanonical initial distribution strategy for classical simulations in strong field physics
}

\author{
F. Mauger ${ }^{1}$, A. Kamor ${ }^{2}$, C. Chandre ${ }^{1}$ and T. Uzer ${ }^{2}$ \\ ${ }^{1}$ Centre de Physique Théorique, CNRS - Aix-Marseille Université, Campus de Luminy, \\ case 907, 13288 Marseille Cedex 09, France \\ 2 School of Physics, Georgia Institute of Technology, Atlanta, GA 30332-0430, USA
}

\begin{abstract}
Classical models in strong field physics typically require a careful selection of initial conditions which correspond accurately to the state selection. We compare two such procedures, the (many) pilot atom technique and the microcanonical strategy. We show that for some range of parameters, the pilot atom strategy may lead to artifacts due to the correlations between the different initial conditions. On the other hand, the microcanonical strategy is, by design, free of such artifacts for all admissible parameters.
\end{abstract}

\section{INTRODUCTION}

Despite their vastly different temporal and spatial scales, celestial mechanics and atomic physics have much in common due to the similarity between the gravitational and Coulomb interactions [1]. As a consequence, many tools of celestial dynamics can be applied to atomic physics and vice versa. The best-known such instance, the Bohr model of the hydrogen atom is, of course, a miniature solar system [2]. It is remarkable that in the almost hundred years that have passed since the inception of this model, analogies between celestial and atomic systems continue to be discovered and exploited. For example, the Restricted Three-Body problem (RTBP) (made up of Sun-Jupiter-Asteroid), is closely related to Rydberg atom ionization in microwave fields [1-3]. The basic theory of chemical reactions, Transition State Theory [4-6], has turned out to be relevant for asteroid capture [7-9] and may one day be useful for the design of spacecraft missions [10]. More recently, the celestial-atomic analogy has been extended to the ionization properties of atoms subjected to strong and short circularly polarized (CP) laser fields [11].

When atomic and molecular systems interact with strong and short laser pulses, many outcomes are possible. One of these, which has attracted a great deal of interest, is correlated double ionization: It gives access to the electronic properties of matter such as experimental imaging of molecular orbitals [12]. Conventionally, for near-infrared linearly polarized laser fields, two main routes to double ionization have been identified [13]. The first one, which is also the most straightforward, is sequential double ionization (SDI) where the two electrons are ionized independently of each other in an uncorrelated way. An alternative route to SDI is the so-called nonsequential double ionization (NSDI) pathway where inter-electronic correlations are deeply involved in the ionization process. The mechanism behind NSDI is now fairly well understood, at least in its broad lines, and follows the recollision scenario [14,15]: First, an electron is ionized by the field, travels in the field absorbing energy, and then, upon reversal of the field direction, it is driven back to the core region where it interacts and exchanges energy with the other electron (recollides). Finally under the impact of the preionized electron, both electrons are ionized in a correlated way. Note that the recollision

This is an Open Access article distributed under the terms of the Creative Commons Attribution License 2.0, which permits unrestricted use, distribution, and reproduction in any medium, provided the original work is properly cited. 


\section{Web of Conferences}

mechanism, by itself, is of great theoretical and experimental interest as one of its byproducts are HHG spectra currently reaching into the XUV and VUV regions [16] providing sub-femtosecond pulses and unprecedented time resolution.

A signature of NSDI (and thus recollision) with linearly polarized (LP) laser fields is the characteristic "knee" shape in the double ionization probability versus laser intensity [13,17]. The curve typically exhibits an enhancement, roughly speaking a bump, in the intermediate range of intensities that departs by several orders of magnitude from what the SDI mechanism predicts. This knee shape has been the first experimental evidence of an alternative route to double ionization from SDI and is now regarded as one of the most dramatic manifestation of electron-electron correlation in nature [13].

Once the recollision mechanism was understood for linear polarization, it was natural to ask whether it persists as the ellipticity of the external field is varied. From the atomic physics point of view, the very same models that explain recollision with linear polarization so well predict its suppression as the ellipticity is increased, and that it would be completely eliminated for circular polarization [14, 18]. On the other hand, from the celestial mechanics point of view, the persistence of recollisions is not much of a surprise since asteroid capture - the counterpart of recollision - is well known and understood by now [1-3]. As a consequence, the question of recollision with CP fields was left open with apparently contradictory experimental results until that is the absence of a knee shape for He and Xe [19,20], which tends to validate the absence of recollision, while a knee shape has been observed for the double ionization of $\mathrm{Mg}$ [21] and nitrogen oxide [22] in CP fields. Recently, it was shown that recollision is indeed possible for circular polarization but it is not accessible to all atoms due to their ground state configurations [11].

Entirely classical interactions turn out to be adequate to generate the strong two-electron correlation needed for double ionization [13,23]. Therefore we work with the classical Hamiltonian model of pseudo-two electron atoms with soft Coulomb potentials [24,25]. We consider a two active electron model atom subjected to a strong and short circularly polarized laser field. In the dipole approximation and considering soft Coulomb potentials [24], the corresponding Hamiltonian reads

$$
\begin{aligned}
\mathcal{H}\left(\mathbf{x}_{1}, \mathbf{x}_{2}, \mathbf{p}_{1}, \mathbf{p}_{2}, t\right)= & \frac{\left|\mathbf{p}_{1}\right|^{2}}{2}+\frac{\left|\mathbf{p}_{2}\right|^{2}}{2}+\frac{1}{\sqrt{\left|\mathbf{x}_{1}-\mathbf{x}_{2}\right|^{2}+b^{2}}}-\frac{2}{\sqrt{\left|\mathbf{x}_{1}\right|^{2}+a^{2}}}-\frac{2}{\sqrt{\left|\mathbf{x}_{2}\right|^{2}+a^{2}}} \\
& +\left(\mathbf{x}_{1}+\mathbf{x}_{2}\right) \cdot f(t) E_{0}\left(\mathbf{e}_{x} \sin \omega t+\mathbf{e}_{y} \epsilon \cos \omega t\right),
\end{aligned}
$$

where $\mathbf{x}_{1}$ and $\mathbf{x}_{2}$ are the positions of the electron (the nucleus is assumed fixed at the origin) in $\mathbb{R}^{d}$ $(d \in\{1,2,3\}), \mathbf{p}_{1}$ and $\mathbf{p}_{2}$ their canonically conjugated momenta, $\mathbf{e}_{x}$ and $\mathbf{e}_{y}$ are the unit vectors in the $x$ - and $y$-directions respectively and $|\cdot|$ and denote the Euclidean norm and scalar product in $\mathbb{R}^{d}$. Unless specified otherwise, atomic units are used throughout the paper. We consider a laser with an envelope $f$, amplitude $E_{0}$, frequency $\omega$ and ellipticity $\epsilon$. For numerical simulations, we consider a trapezoid shape $f$ with two laser cycle ramp up and down and four laser cycle constant plateau. The frequency is set to $\omega=0.0584$, corresponding to a $780 \mathrm{~nm}$ wavelength. Linear polarization corresponds to $\epsilon=0$ and circular polarization to $\epsilon=1$.

Once the model atom is chosen, i.e., for a given value of ground state energy $\mathcal{E}_{g}$ (defined as the sum of the first and second ionization potentials [26]), the softening parameters ( $a$ and $b$ ) have to be determined such that Hamiltonian (1) models the atom well. For the actual selection of the softening parameters we consider the two following constraints:

- Without laser excitation, the atom must be "stable", or, in other words, the model must not allow for self-ionization: We impose the ground state energy surface $\left\{\mathcal{H}=\mathcal{E}_{g}\right\}$ to be bounded in phase space for $E_{0}=0$.

- One should be able to actually generate initial conditions with energy $\mathcal{E}_{g}$ : The ground state energy surface must not be empty $\left\{\mathcal{H}=\mathcal{E}_{g}\right\} \neq \varnothing$. 


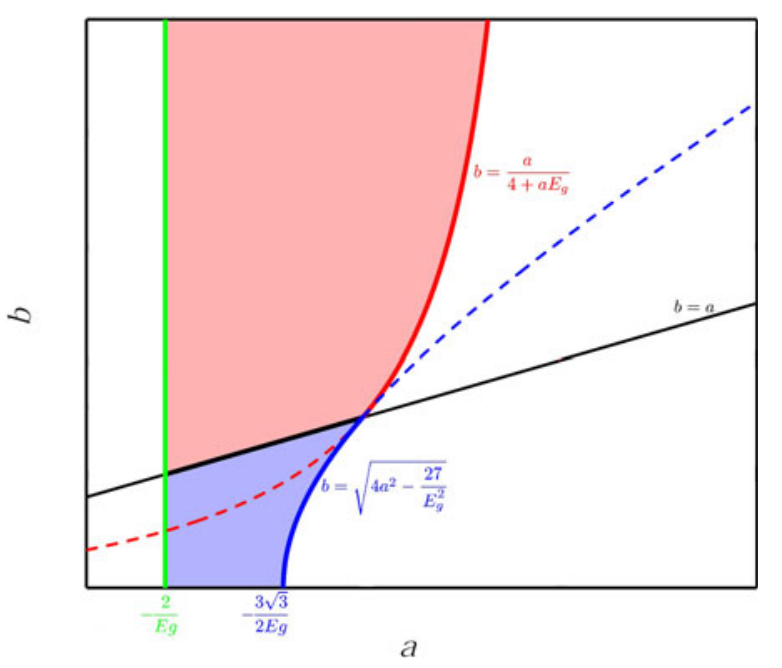

Figure 1. Accessible range of softening parameters (colored area) for a given atom (i.e., ground state energy $\mathcal{E}_{g}$ ) in the $(a, b)$ parameter plane.

By definition, the ground state energy is negative $\left(\mathcal{E}_{g}<0\right)$. As a consequence, the first constraint (no self-ionization) is equivalent to

$$
-\frac{2}{\mathcal{E}_{g}}<a
$$

Note that this constraint only involves the electron-nucleus softening parameter. On the other hand, the second constraint (that the ground state energy surface is not empty), involves the relative magnitude of both $a$ and $b$. We define the two electron potential as

$$
V\left(\mathbf{x}_{1}, \mathbf{x}_{2}\right)=-\frac{2}{\sqrt{\left|\mathbf{x}_{1}\right|^{2}+a^{2}}}-\frac{2}{\sqrt{\left|\mathbf{x}_{2}\right|^{2}+a^{2}}}+\frac{1}{\sqrt{\left|\mathbf{x}_{1}-\mathbf{x}_{2}\right|^{2}+b^{2}}} .
$$

The minimum potential energy is reached when each electron is located on a symmetric (mirror) position about the nucleus at

$$
\left|\mathbf{x}_{1,2}\right|=\sqrt{\frac{\max \left(a^{2}-b^{2}, 0\right)}{3}} .
$$

As a consequence, in order to have a non-empty ground state energy surface, the softening parameters must satisfy

$$
b>\frac{a}{4+a \mathcal{E}_{g}} \quad \text { for } a \leq b,
$$

and

$$
b>\sqrt{4 a^{2}-\frac{27}{\mathcal{E}_{g}^{2}}} \text { otherwise. }
$$

We give an illustration of the range of accessible values for the softening parameters $(a, b)$ in Fig. 1 .

For a given atom, we select the electron-nucleus softening parameter $a$ such that all values of $b$ are admissible, i.e.,

$$
-\frac{2}{\mathcal{E}_{g}}<a<-\frac{3 \sqrt{3}}{2 \mathcal{E}_{g}}
$$




\section{Web of Conferences}

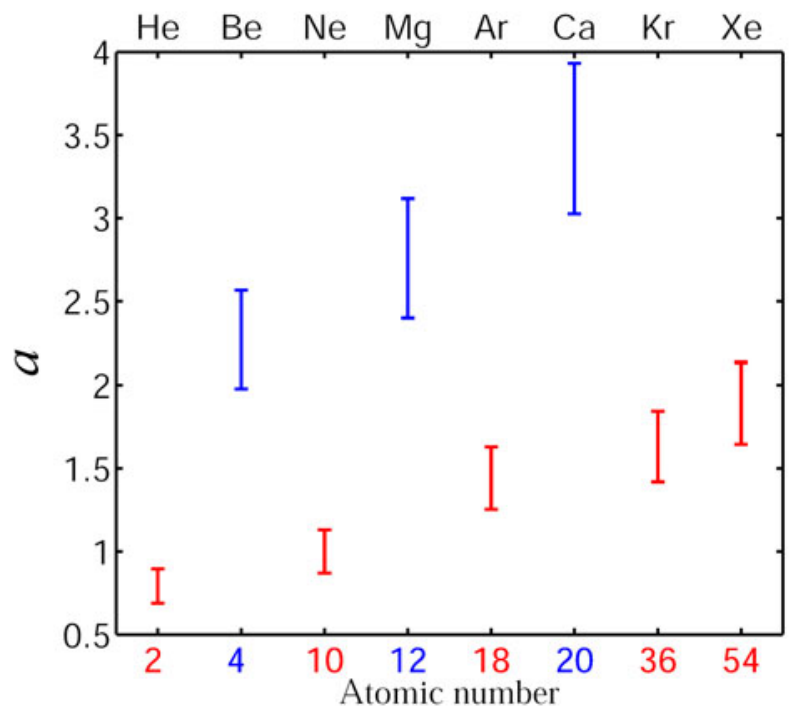

Figure 2. Accessible range of electron-nucleus softening parameter $a$ (see text) for the first rare gas and alkaline earth elements.

and we usually set the electron-electron softening parameter to unity $(b=1)$. In figure 2 we display the corresponding range of parameter $a$ for commonly used atoms.

The softening parameter $a$ enables one to model different atoms while, the parameter $b$ controls the collision strength of the two electron when they pass by each other. As a consequence, one can expect a different organization of the dynamics depending on the chosen parameters, for a given atom (i.e., a given $a$ but different values of $b$ ). In figure 3 we display several Poincaré sections [27] for Hamiltonian (1) with one spatial dimension and without laser excitation $\left(E_{0}=0\right)$. For each panel, we consider the Poincaré section of equation

$$
x_{2}=-\sqrt{\frac{\max \left(a^{2}-b^{2}, 0\right)}{3}}, \quad \text { and } \quad d_{t} x_{2}>0 .
$$

The figures highlight two main observations:

- For a given atom (i.e., chosen $a$ and $\mathcal{E}_{g}$ ), the topology of the accessible part of phase space to the field-free dynamics varies significantly. In particular, we note that the neighborhood of the nucleus is not always accessible to the dynamics.

- Varying the softening parameters changes significantly the organization of the dynamics, for instance

- For $a=3$ and $b=0.1$ (lower left panel) we observe a mostly regular dynamics, where phase space is almost completely foliated with invariant structures.

- For $a=1$ and $b=0.1$ (upper left panel) we observe a mixed dynamics, with coexistence of chaotic seas with regular regions.

- For $a=1.5$ and $b=1$ (central panel) we observe a mostly chaotic dynamics, with a single chaotic sea and barely any invariant structures.

As we shall see in what follows, this observation has a concrete consequence in the way one chooses initial condition for a statistical analysis of the dynamics.

The limit of a large electron-electron softening parameter $(b)$ corresponds to the uncorrelated dynamics [28]. It has a limited physical interest such that we do not comment more on it. On the other 


\section{UVX 2012}
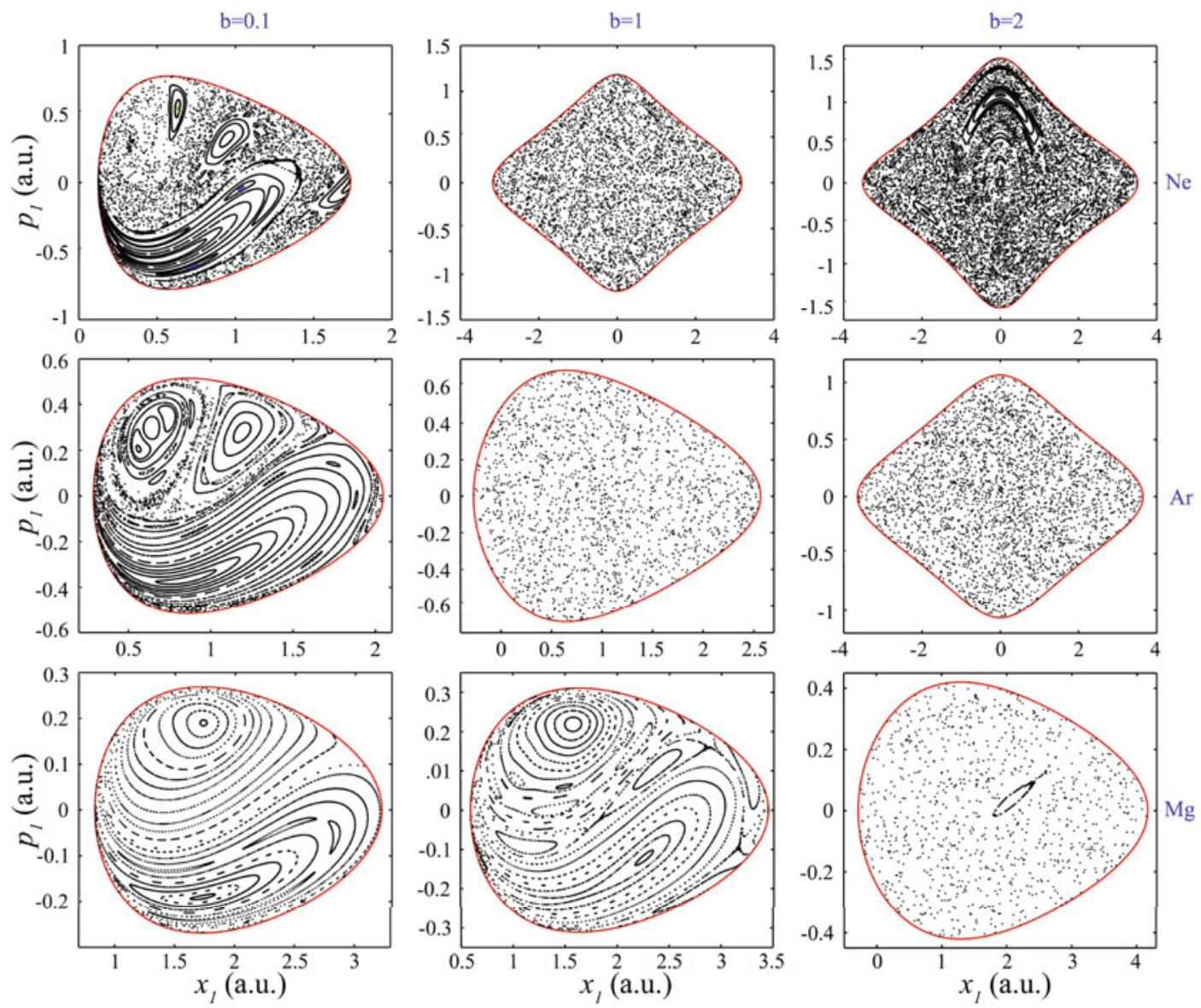

Figure 3. Poincaré sections for Hamiltonian (1) with one spatial dimension. For each panel, we consider the section of equation $x_{2}=-\sqrt{\max \left(a^{2}-b^{2}, 0\right) / 3}, d_{t} x_{2}>0$. The first row corresponds to neon $\left(a=1, \mathcal{E}_{g}=-2.3\right)$, the second to $\arg$ on $\left(a=1.5, \mathcal{E}_{g}=-1.6\right)$ and the third to magnesium $\left(a=3, \mathcal{E}_{g}=-0.83\right)$ [26]. The first column corresponds to $b=0.1$, the second to $b=1$ and the third to $b=2$. Colored areas in the upper left panel correspond to the initial set visited by the pilot atoms considered in Fig. 5.

hand, the limit of a small electron-electron softening parameter $(b)$ forces the electrons to stay away from each other.

\section{INFLUENCE OF THE CHOICE OF INITIAL CONDITIONS ON DOUBLE IONIZATION YIELDS}

Several methods have been considered to generate initial conditions in the ground state energy for classical simulations as given by Hamiltonian (1). In this section, we consider and compare two of them. The first one, called the (many) pilot atom strategy, takes advantage of the property of conservation of the energy for autonomous Hamiltonian system. On the other hand, a microcanonical initial distribution can be considered using a rejection sampling method.

The pilot atom strategy [23, 25,29-35] (first introduced in Ref. [36] for Rydberg atoms) first chooses a point on the ground state energy surface. Then, using the field-free dynamics [Hamiltonian (1) with $E_{0}=0$ ], the corresponding trajectory is propagated in time and points (in phase space) are picked 


\section{Web of Conferences}

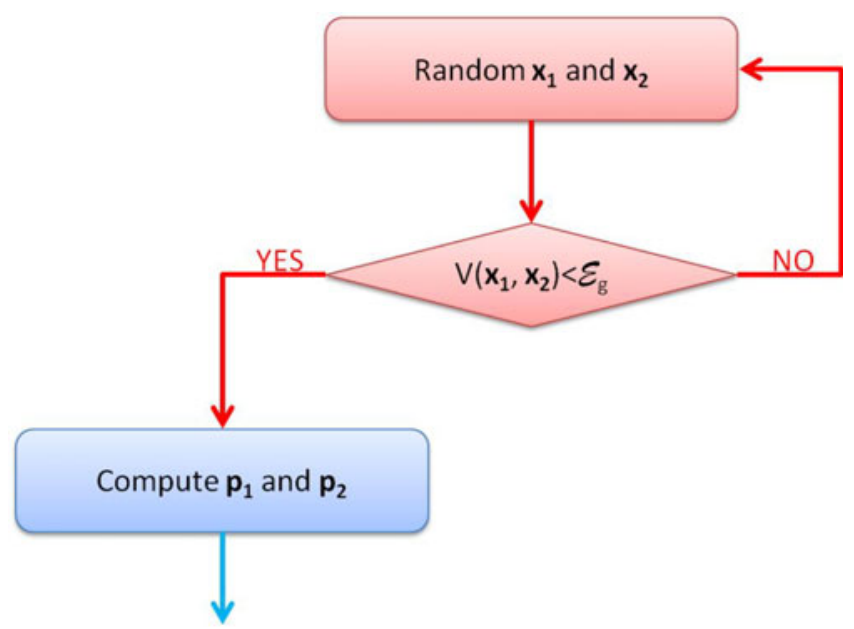

Random initial condition on the ground state energy

Figure 4. Sketch of algorithm used to compute the microcanonical initial distribution on the ground state energy surface. The diamond block labels a logical test.

along the pilot trajectory. Some variations of the strategy use several pilot trajectories to generate the pool of initial conditions. For the numerical simulations we consider in this section, we consider a single sampling trajectory and initial conditions are chosen every 10 a.u. (in time). Since the fieldfree dynamics corresponds to an autonomous Hamiltonian system, the total energy is conserved by the dynamics such that all the points along the pilot trajectory have the same energy, corresponding to the ground state energy.

The microcanonical strategy $[28,37]$ decomposes Hamiltonian (1) with $E_{0}=0$ into a kinetic energy $\mathcal{T}$ plus potential $V$ where

$$
\mathcal{T}=\frac{\left|\mathbf{p}_{1}\right|^{2}}{2}+\frac{\left|\mathbf{p}_{2}\right|^{2}}{2}
$$

and $V$ is given by Eq. (2), such that $\mathcal{H}=\mathcal{T}+V$. First we generate a uniform distribution over the subspace $\mathcal{V}=\left\{\left(\mathbf{x}_{1}, \mathbf{x}_{2}\right) \in \mathbb{R}^{2 d}\right.$ s.t. $\left.V\left(\mathbf{x}_{1}, \mathbf{x}_{2}\right) \leq \mathcal{E}_{g}\right\}$. By hypothesis, the subset $\mathcal{V}$ is bounded and we find convenient to use a rejection sampling method [38] to obtain a uniform distribution: We consider a distance $d_{\text {crit }}$ such that $\mathcal{V} \subset\left[-d_{\text {crit }}, d_{\text {crit }}\right]^{2 d}$. Then, we pick up an initial position for the two electrons $\left(\mathbf{x}_{1}, \mathbf{x}_{2}\right)$ in $\left[-d_{\text {crit }}, d_{\text {crit }}\right]^{2 d}$ with a uniform distribution. If $V\left(\mathbf{x}_{1}, \mathbf{x}_{2}\right)>\mathcal{E}_{g}$, a new position is chosen randomly with the same uniform distribution (actually we iterate the process until we find a suitable position). Finally, the momenta $\mathbf{p}_{1}$ and $\mathbf{p}_{2}$ are chosen with a uniform distribution over the sphere (in $\mathbb{R}^{2 d}$ ) of radius $\sqrt{2\left(\mathcal{E}_{g}-V\left(\mathbf{x}_{1}, \mathbf{x}_{2}\right)\right)}$. Note that the final step is straightforward (it can be performed by choosing randomly $2 d-1$ angles). We give a sketch of the algorithm we use to generate a microcanonical initial distribution on the ground state energy surface in Fig. 4.

In figure 5 we compare the statistical results obtained with a microcanonical distribution (continuous curve) and different pilot atom strategies (markers). A first surprise is that, depending on the way the pool of initial conditions is generated, double ionization curves depart dramatically from each other showing (up to) orders of magnitude of difference. In addition, the pilot atom method exhibits a significant sensitivity to the selected pilot trajectory.

Going back to the ionization probability with the pilot atom strategy, by looking for instance at the red squares in Fig. 5 (right panel), one would have missed the existence of the knee and thus conclude the absence of recollisions with CP fields and $\mathrm{Mg}$. To some extent the same conclusions would be reached 

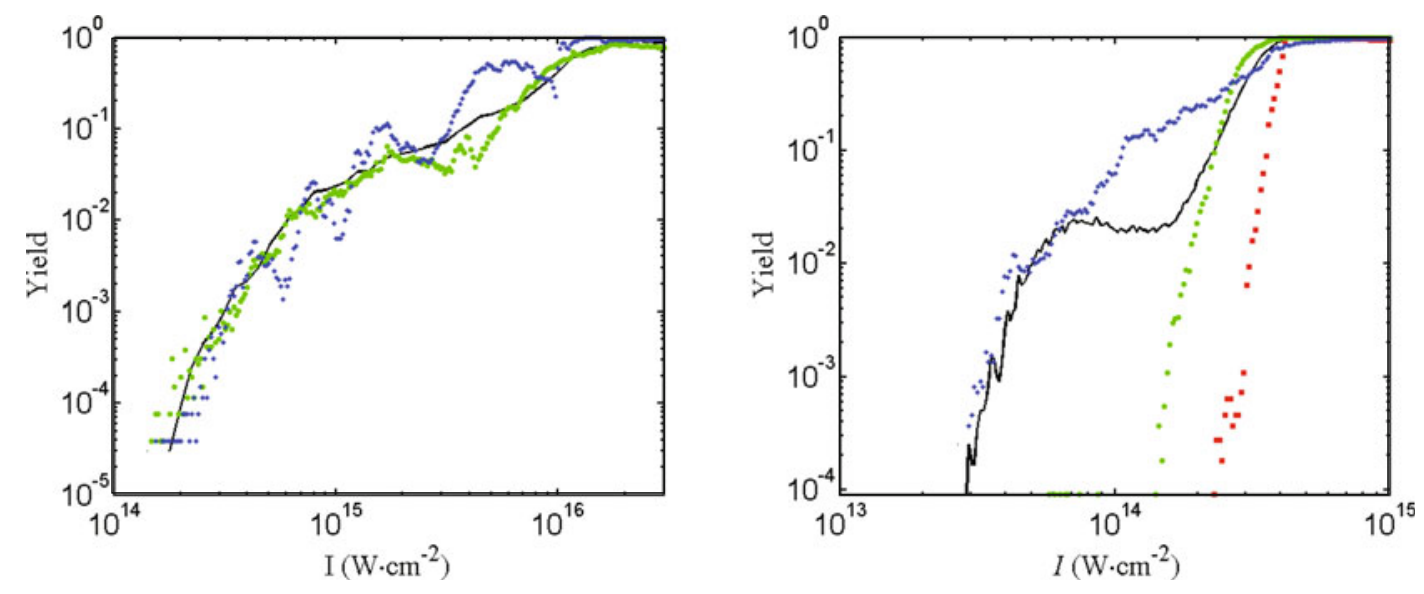

Figure 5. Double ionization probability as a function of the laser intensity for Hamiltonian (1) with one (left panel) and two (right) spatial dimensions. In both panels we consider a trapezoid shape for the laser with two laser cycle ramp up and down and four laser cycle plateau. The continuous line corresponds to a microcanonical initial distribution and colored markers correspond to various pilot atom strategies (see text). In the left panel, we consider a neon model $\left(a=1, b=0.1\right.$ and $\left.\mathcal{E}_{g}=-2.3\right)$ with linear polarization and in the right panel we consider magnesium ( $\left.a=2, b=1, \mathcal{E}_{g}=-0.83\right)$ with circular polarization. The initial set for the pilot atom strategies is displayed in Fig. 3 using the same color code (upper left panel).

with green dots in the same figure. On the other hand, considering the microcanonical distribution, we observe a well-defined knee. Finally, looking at the blue diamonds (yet another pilot atom), we see that the double ionization probability is significantly overestimated in a wide range of intensities.

The rationale behind the discrepancies obtained with the (many) pilot atom strategy can be explained with the dynamical organization of the accessible phase space of the field-free dynamics. In particular for some set of parameters, phase space is partially (or even almost entirely) covered by regular invariant structures. This has a direct impact on the way initial conditions are generated for a statistical analysis of the dynamics (including the excited dynamics).

\section{CONCLUSION}

For (even partially) regular dynamics, the many pilot atom method takes the risk of having initial condition that do not cover the entire accessible ground state energy surface, with a strong correlation between the different initial conditions (if the pilot trajectory is stuck on an invariant torus for instance). It is what is observed in Fig. 5 (left panel), where the pilot trajectory is confined to a small portion of the accessible phase space, as shown in Fig. 3 (colored areas in the upper left panel). On the other hand, for a chaotic dynamics, a typical trajectory fills very quickly the whole accessible region in phase space. In this case the two kinds of initial sets lead to approximately similar results if the integration time as well as the number of points are sufficiently large [28]. In any case, in order to avoid artifacts due correlation effects between the initial conditions, a microcanonical strategy is best to consider.

\section{Acknowledgments}

The research leading to these results has received funding from the People Programme (Marie Curie Actions) of the European Union’s Seventh Framework Programme FP7/2007-2013/ under REA grant agreement n 94974. F.M. acknowledges financial support from the Fulbright program. This work is partially funded by NSF. 
Web of Conferences

\section{References}

[1] E. Lee, A. F. Brunello, C. Cerjan, T. Uzer, and D. Farrelly, in The Physics and Chemistry of Wave Packets, edited by J. A. Yeazell and T. Uzer (John Wiley, New York, 2000), p. 95

[2] E. Lee, D. Farrelly, and T. Uzer, Optics Express 1, 221 (1997)

[3] T. Uzer, E. A. Lee, D. Farrelly, and A. F. Brunello, Contemp. Phys. 41, 1 (2000)

[4] J. C. Keck, Adv. Chem. Phys. 13, 85 (1967)

[5] T. Uzer, C. Jaffé, J. Palacián, P. Yanguas, and S. Wiggins, nonlinearity 15, 957 (2002)

[6] C. Jaffé, S. Kawai, J. Palacián, P. Yanguas, and T. Uzer, Adv. Chem. Phys. 130A, 171 (2005)

[7] C. Jaffé, S. D. Ross, M. W. Lo, J. Marsden, D. Farrelly, and T. Uzer, Phys. Rev. Lett. 89, 011101 (2002)

[8] C. Jaffé and T. Uzer, Ann. N.Y. Academy of Sciences 1017, 39 (2004)

[9] C. Jaffé and T. Uzer, Nucl. Phys. A 737, 125 (2004)

[10] W. Koon, M. Lo, J. Marsden, and S. Ross, Chaos 10, 427 (2000)

[11] F. Mauger, C. Chandre, and T. Uzer, Phys. Rev. Lett. 105, 083002 (2010)

[12] S. Haessler, J. Caillat, W. Boutu, C. Giovanetti-Teixeira, T. Ruchon, T. Auguste, Z. Diveki, P. Breger, A. Maquet, B. Carre, et al., Nature Physics 6, 200 (2010)

[13] W. Becker and H. Rottke, Contemporary Physics 49, 199 (2008)

[14] P. B. Corkum, Phys. Rev. Lett. 71, 1994 (1993)

[15] K. J. Schafer, B. Yang, L. F. DiMauro, and K. C. Kulander, Phys. Rev. Lett. 70, 1599 (1993)

[16] C. Figueira de Morisson Faria and X. Liu, J. Modern. Opt. 58, 1076 (2011)

[17] B. Walker, B. Sheehy, L. F. DiMauro, P. Agostini, K. J. Schafer, and K. C. Kulander, Phys. Rev. Lett. 73, 1227 (1994)

[18] P. B. Corkum, Phys. Today 64, 36 (2011)

[19] B. Walker, E. Mevel, B. Yang, P. Breger, J. P. Chambaret, A. Antonetti, L. F. DiMauro, and P. Agostini, Phys. Rev. A 48, R894 (1993)

[20] D. N. Fittinghoff, P. R. Bolton, B. Chang, and K. C. Kulander, Phys. Rev. A 49, 2174 (1994)

[21] G. D. Gillen, M. A. Walker, and L. D. Van Woerkom, Phys. Rev. A 64, 043413 (2001)

[22] C. Guo and G. N. Gibson, Phys. Rev. A 63, 040701 (2001)

[23] P. J. Ho, R. Panfili, S. L. Haan, and J. H. Eberly, Phys. Rev. Lett. 94, 093002 (2005)

[24] J. Javanainen, J. H. Eberly, and Q. Su, Phys. Rev. A 38, 3430 (1988)

[25] R. Panfili, J. H. Eberly, and S. L. Haan, Opt. Express 8, 431 (2001)

[26] Atomic data are taken from the NIST website http://physics.nist.gov/PhysRefData/ Handbook/periodictable.htm

[27] P. Cvitanović, R. Artuso, R. Mainieri, G. Tanner, and G. Vattay, Chaos: Classical and Quantum (Niels Bohr Institute, Copenhagen, 2008), http: //ChaosBook.org

[28] F. Mauger, C. Chandre, and T. Uzer, J. Phys. B. 42, 165602 (2009)

[29] X. Wang and J. Eberly, Phys. Rev. Lett. 103, 103007 (2009)

[30] J. Guo and X.-s. Liu, Phys. Rev. A 78, 013401 (2008)

[31] V. Gridchin, Laser Phys. 15, 456 (2005)

[32] S. L. Haan, J. C. Cully, and K. Hoekema, Opt. Express 12, 4758 (2004)

[33] D. Bauer, Phys. Rev. A 56, 3028 (1997)

[34] P. J. Ho and J. H. Eberly, Phys. Rev. Lett. 95, 193002 (2005)

[35] P. J. Ho, Phys. Rev. A 72, 045401 (2005)

[36] R. Abrines and I. C. Percival, Proc. Phys. Soc. London 88, 861 (1966)

[37] F. Mauger, C. Chandre, and T. Uzer, Phys. Rev. Lett. 102, 173002 (2009)

[38] J. von Neumann, Nat. Bureau Standards 12, 36 (1951) 